\title{
CRONOLEX: sistema para la representación dinámica de cuerpos legales
}

\author{
Javier de Andrés Rivero \\ Rafael Hernández Marín \\ Universidad de Murcia (España)
}

\section{Resumen}

En este artículo se presenta la arquitectura de un sistema para la recuperación del derecho español de forma eficiente, que utiliza como base XML y registra metainformación junto al propio cuerpo legal. Esto permite definir una base de datos consolidada para recuperar el estado de un cuerpo legal en una fecha concreta. La estructura XML diseñada es suficientemente flexible para representar cualquier tipo de cuerpo legal existente en España.

Palabras clave: XML. Recuperación. Derecho vigente. Metainformación.

\section{Abstract}

In this paper an architecture of a system is presented. This architecture has been designed for the efficient retrieval of Spanish laws, based on the metainformation structured in XML, to allow the definition of a consolidated database to retrieve the state of a legal norm in a certain date. The XML structure is sufficiently flexible to be able to represent all the types of legal documents that exist in Spain.

Keywords: XML. Retrieval. Law in force. Metadata.

\section{Motivación}

Es muy común, en la legislación española, que desde la publicación de un cuerpo legal aparezcan otros que introduzcan modificaciones en el texto de ese cuerpo legal. Cada una de estas modificaciones origina, por así decirlo, una nueva versión de ese texto, de manera que desde la publicación de un cuerpo legal hasta una fecha concreta se pueden tener diferentes versiones del mismo. Hay que destacar que estas nuevas "versiones" son documentos meramente virtuales, ya que no existen textos refundidos con el texto resultante.

Esto, que puede parecer fácil de manejar, se hace difícilmente manejable para cuerpos legales que se modifican continuamente con el paso de los años. Un ejemplo de esto se puede ver en el Código Penal español, que desde su publicación en 1994 hasta la fecha actual ha tenido muchas versiones debido a la gran cantidad de

Scire. 15 : 1 (en.-jun. 2009) 133-147. ISSN 1135-3716. 
cuerpos legales que se han publicado y han modificado el texto original. En la actualidad, las editoriales privadas, partiendo de ese texto original y de los cuerpos legales modificantes posteriores, redactan esos documentos virtuales de forma manual (cortando, copiando y pegando texto).

El objetivo que se ha perseguido con este proyecto es elaborar un programa informático que $a$ ) redacte de forma automática esos documentos virtuales, que actualmente son elaborados de forma manual, y $b$ ) nos diga cuál de esos documentos virtuales, que son versiones diferentes del Código Penal, es el que pertenece al derecho en cualquier fecha comprendida entre 1994 (año de publicación del texto original del código) y el día de hoy.

\section{Introducción}

Como se ha podido ver en la el apartado dedicado a la motivación, lo que se ha perseguido en el proyecto Cronolex es la creación de un sistema informático de gestión de textos jurídicos con vistas a la elaboración de una base de datos consolidada que permita la recuperación automática del derecho en vigor. Este sistema informático podrá ser utilizado en los procesos de creación normativa por las distintas entidades con competencia en el ámbito de la producción de derecho, y es aplicable a cualquier sector del ordenamiento jurídico.

El objetivo es generar una herramienta que permita la localización de información relevante para el interesado en un determinado instante y en un ámbito concreto, que en nuestro caso se identifica con un ordenamiento jurídico nacional. De acuerdo con este objetivo, se ha optado por organizar y estructurar los textos jurídicos que han de ser incorporados a la base de datos, empleando para ello las ventajas que proporciona la tecnología XML, de modo que se obtenga un conjunto uniforme de datos relacionados entre sí.

Mediante el uso de XML se puede asociar las diferentes partes del texto jurídico con un núcleo de información relevante que, por un lado, facilita diversos tipos de búsquedas y, por otro, permite la interacción entre partes del mismo texto jurídico o entre partes de diversos textos jurídicos integrados en la misma base de datos. Esto último es especialmente útil en nuestro caso, puesto que la organización del material normativo depende en gran parte de las relaciones entre los enunciados que forman parte de los textos jurídicos.

De las posibles relaciones que se operan entre los componentes de los textos jurídicos son especialmente interesantes aquellas que causan la modificación de esos mismos componentes provocando un cambio constante en la información jurídica relevante. Así pues, la búsqueda de la información jurídica no se lleva a cabo respecto a un conjunto sistematizado y estático de datos, sino respecto a un conjunto de datos que se modifican con la introducción de nuevos datos. 
De este modo, considerado el ordenamiento jurídico en momentos (días) distintos, podemos observar que su contenido también es distinto. Es posible que aparezcan cuerpos legales nuevos que el día anterior no existían, o puede que cuerpos legales que el día anterior pertenecían al ordenamiento jurídico ya no sigan correspondiendo a dicho ordenamiento. Incluso puede variar el contenido de un mismo cuerpo legal que pertenece al derecho en días distintos, porque algunos de sus enunciados han sido derogados, incorporados o sustituidos.

Nuestro sistema pretende reflejar esa realidad dinámica del ordenamiento jurídico, con vistas a facilitar la localización de la información relevante en cada momento para el interesado. Es decir, permitir la búsqueda de los enunciados pertenecientes al derecho tal y como se encuentran en el momento de la búsqueda o bien en cualquier otro momento anterior solicitado por el interesado.

La obtención de este resultado pasa por especificar en qué consisten las diversas operaciones de modificación normativa (derogación, incorporación y sustitución), de modo que el sistema esté en condiciones de reconocer cuándo se producen y a qué textos afectan. Para obtener dicho resultado se requiere un sistema que permita operar de manera automática con los cambios normativos teniendo en cuenta la información suministrada por los propios documentos que integran la base de datos.

Se debe tener en cuenta que los cambios efectuados en los cuerpos legales no se reflejan en la creación de ningún texto real. La operación, pues, de ofrecer los cuerpos legales actualizados se ha de hacer a través de la elaboración, por el sistema, del texto jurídico virtual tal y como sería obtenido de la aplicación por el intérprete de las operaciones modificatorias indicadas por los cuerpos legales modificantes.

Trabajando sobre la base de iniciativas precedentes, nos interesaba especialmente destacar los aspectos temporales que rodean las modificaciones normativas y asegurar que el programa responda adecuadamente al tratamiento teórico de esos aspectos temporales.

La gestión automatizada de las modificaciones normativas solamente dará resultados aceptables si funciona de acuerdo con los criterios temporales conforme a los que se van produciendo tales modificaciones. Es decir, el programa no solo debe reconocer que un determinado enunciado jurídico ha sido derogado o incorporado, sino que también ha de mostrar los efectos de esa modificación en el momento adecuado. De este modo, cualquier consulta localizada en un día anterior a la producción del efecto tiene que ofrecer como resultado el texto jurídico no modificado, mientras que una consulta localizada en el día en que el efecto se produce o en otro posterior debe mostrar el texto tal y como queda tras la modificación.

Para lograr este objetivo es necesario especificar claramente los criterios que sirven para encuadrar los límites temporales de la pertenencia de un texto al Scire. $15: 1$ (en.-jun. 2009) 133-147. ISSN 1135-3716. 
ordenamiento jurídico, lo que nos permite considerar a ese texto como enunciado jurídico en el intervalo comprendido entre dichos límites. Los criterios que han sido empleados en el desarrollo de nuestro programa están tomados del análisis realizado sobre el intervalo de validez de los enunciados jurídicos (Hernández Marín, 2002). Por intervalo de validez se entiende, precisamente, ese período de tiempo en el que un texto pertenece al ordenamiento jurídico.

De acuerdo con estos criterios, los límites temporales del intervalo de validez de un enunciado jurídico se encuentran en el día de la publicación (límite inicial) y el de la derogación (límite final). Por lo tanto, la pertenencia de un texto al derecho queda enmarcada en el período de tiempo comprendido entre el día de su publicación (incluido) y el de su derogación (no incluido).

La determinación del día de la publicación normalmente no es problemática, puesto que viene identificado con la fecha del diario oficial en el que el texto aparece. Los mayores problemas vienen a la hora de determinar la fecha de la derogación.

Lo que se va indicar a continuación son los criterios utilizados para determinar el día de una derogación expresa formal, es decir, aquella que se produce por medio de una disposición derogatoria formal.

El día de la derogación del cuerpo legal se corresponde con el tiempo del efecto de la disposición derogatoria.

El tiempo del efecto de la disposición derogatoria será el día señalado en la propia disposición derogatoria para la producción de dicho efecto. Si la disposición derogatoria no contiene indicaciones sobre su tiempo de efecto (como suele ser habitual), su tiempo de efecto coincidirá con la fecha de entrada en vigor del cuerpo legal al que la disposición derogatoria pertenece.

La derogación solo produce efectos sobre enunciados que no hayan sido derogados previamente, por lo cual el texto derogado debe ser todavía un enunciado jurídico el día anterior a aquel que corresponde al tiempo del efecto de la disposición derogatoria. De otro modo, el día de la derogación de ese enunciado jurídico será un día previo, correspondiente al tiempo del efecto de otra disposición derogatoria.

La disposición derogatoria debe pertenecer al ordenamiento jurídico el día de la derogación, puesto que si previamente ha sido derogada no podrá producir el efecto previsto. Esto supone que el sistema no tendrá en cuenta la derogación de un enunciado jurídico si comprueba que la disposición derogatoria ha sido, a su vez, derogada cualquier día anterior al de la derogación.

\section{Arquitectura del sistema}

Para la resolución del problema que se plantea se ha diseñado la siguiente arquitectura: 


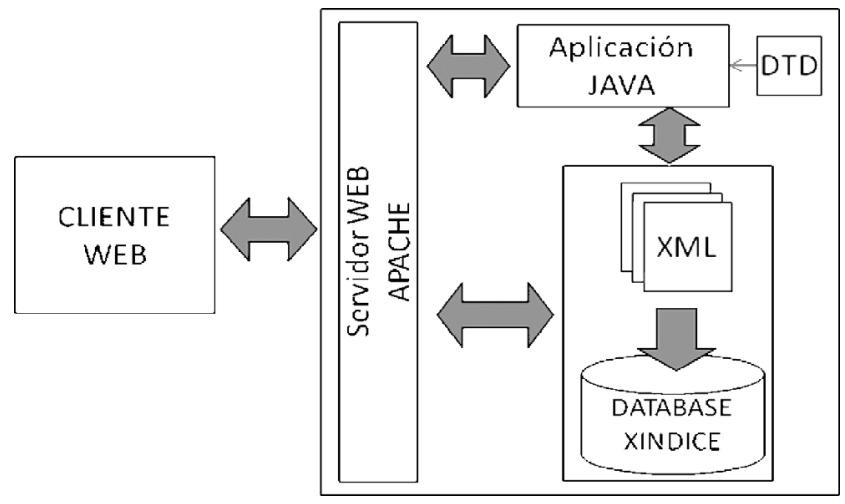

Figura 1. Arquitectura realizada.

Como se puede observar en la figura 1 , se ha diseñado una arquitectura compuesta por: $a$ ) DTD, creado con una estructura flexible para representar cualquier rango de un cuerpo legal; $b$ ) documentos XML, que representan los cuerpos legales y que siguen el DTD creado; $c$ ) base de datos nativa XML, donde se van a guardar los documentos XML (se ha elegido XINDICE como gestor de base de datos nativa XML); $d$ ) módulos JAVA, que se van a encargar de procesar los documentos XML (se explican en los puntos siguientes); $e$ ) servidor web, donde se instala tanto la base de datos nativa XML como las aplicaciones web que utilizan las librerías JAVA para procesar los XML; $f$ ) cliente web: navegador de Internet con el cual ejecutar las aplicaciones web.

Para gestionar todo el ciclo de vida de un cuerpo legal se han realizado los siguientes módulos: módulo de edición de cuerpos legales, módulo de base de datos, módulo de consolidación, módulo de búsqueda y módulo web.

\subsection{Módulo de edición de los cuerpos legales}

Este módulo (o parte del proyecto) es el encargado de transformar el texto original del cuerpo legal en un documento XML con la estructura necesaria. Su tarea más importante (y piedra angular del proyecto) fue desarrollar una estructura XML lo suficientemente flexible como para definir todos los tipos de cuerpos legales que hay en la legislación española. Una vez definida la estructura, esta fue descrita en un DTD (Document Type Definition).

La característica más importante de esta estructura XML es que junto al texto del cuerpo legal original se guarda toda la metainformación necesaria para indicar las modificaciones que otros cuerpos legales van realizando sobre el original a lo largo del tiempo. La estructura XML diseñada tiene dos grandes partes: la metainformación y el texto del cuerpo legal.

Scire. $15: 1$ (en.-jun. 2009) 133-147. ISSN 1135-3716. 
El módulo actual de edición de cuerpos legales se basa en una aplicación web en la que se pueden ir introduciendo todos los datos de un cuerpo legal en una serie de formularios preparados para tal efecto.

En estos momentos estamos investigando para automatizar esta tarea a partir de un transformador automático que vaya procesando el cuerpo legal (en formato digital) y creando la estructura XML que le corresponda. Cuando el transformador termine indicará todo lo que no ha podido procesar para que un experto en derecho indique (según unas reglas) cómo hay que transformar ese cuerpo legal, de modo que el transformador sepa cómo ha de funcionar la próxima vez que se encuentre con ese problema.

\subsubsection{Metainformación}

La información que se guarda dentro de la etiqueta $<$ MetaInformación $>$ no forma parte del texto del cuerpo legal, sino que es información que identifica y describe el cuerpo legal. Se pueden distinguir dos partes dentro de la metainformación: a) una primera parte donde se incluye toda la información necesaria para el cuerpo legal (información obligatoria); esta información la tendrán todos los cuerpos legales e incluye datos como la fecha de publicación, el rango del cuerpo legal, el identificador dentro del sistema...; $b$ ) una segunda parte donde se incluye información opcional, como podría ser el nombre oficioso del cuerpo legal.

Dentro de la segunda parte se incluyen las etiquetas referentes a las modificaciones que haya experimentado el texto del cuerpo legal desde la fecha de su publicación, modificaciones que pueden ser de tres tipos: supresión de texto (bien por derogación, bien por anulación), incorporación o adición de texto nuevo, y sustitución de un texto por otro. Es decir, esta segunda parte es la que se actualiza cuando el cuerpo legal es modificado por otro.

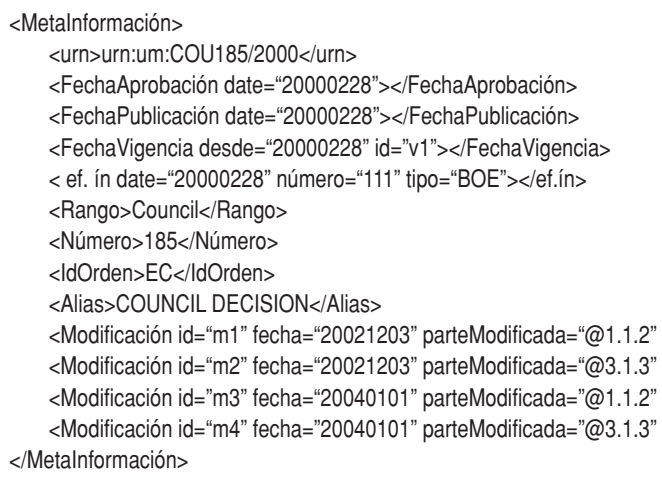

xlink: ef.= "urn:um:COU954/2002@1.1.1"/> xlink: ef.= "urn:um:COU954/2002@1.1.2"/> xlink: ef.= "urn:um:COU161/2004@1.1.1"/> xlink: ef.= "urn:um:COU161/2004@1.1.2"/>

Figura 2. Ejemplo de metainformación. 
Las modificaciones se registran con las etiquetas $<$ Derogación $>$, $<$ Anulación $>$, $<$ Incorporación $>$ y $<$ Sustitución $>$. Un ejemplo de la metainformación de un cuerpo legal es el siguiente:

\subsubsection{Texto del cuerpo legal}

La parte del XML donde se guarda el texto del cuerpo legal incluye etiquetas para registrar todas las partes que puede tener dicho texto en la legislación española. Son estas:

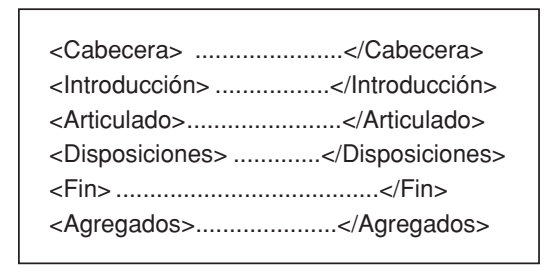

Figura 3. Etiquetas que representan el texto del cuerpo legal.

A continuación se detalla cada una de las etiquetas:

Cabecera. Es la primera etiqueta que guarda texto del cuerpo legal; en ella se guarda la cabecera (o título) de dicho cuerpo legal.

Introducción. Parte donde se estructura la introducción del cuerpo legal (también llamada Preámbulo). Puede estar compuesta por las "Exposiciones de motivos" o puede ser solo un texto introductorio. Esta etiqueta no es obligatoria, ya que no todos los cuerpos legales tienen introducción.

Articulado. Parte de la estructura que representa los artículos que contiene el cuerpo legal. Esta etiqueta puede estar estructurada del siguiente modo:

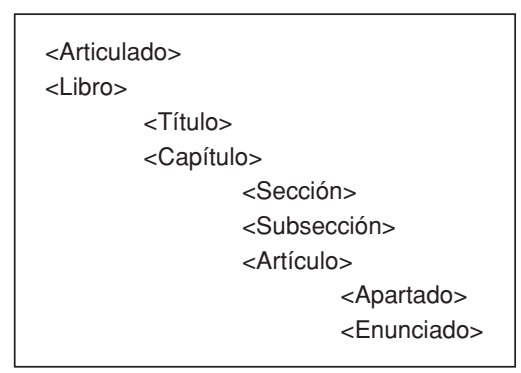

Figura 4. Partes del articulado.

Scire. $15: 1$ (en.-jun. 2009) 133-147. ISSN 1135-3716. 
Todas estas divisiones no son obligatorias para todos los cuerpos legales. Algunos están estructurados con todas estas divisiones, otros solo se dividen en algunas de ellas y otros no se dividen en ninguna, sino que están estructurados únicamente en los artículos.

Disposiciones. Parte que representa las disposiciones que tiene un cuerpo legal. Estas pueden ser "Adicionales", "Transitorias", "Derogatorias" y "Finales".

Fin. Parte final del cuerpo legal; incluye la firma y fecha del cuerpo legal.

Agregados. Esta parte representa cualquier texto que es incluido al final del cuerpo legal.

\subsubsection{Otras etiquetas}

Las partes definidas anteriormente son comunes para todos los cuerpos legales, tanto los originales como los modificantes. Sin embargo, un cuerpo legal modificante incluye una serie de etiquetas adicionales que indican un cambio en el cuerpo legal modificado. Hay que recordar que hay tres tipos de modificaciones: sustituciones, incorporaciones y supresiones (bien por anulaciones o bien por derogaciones).

Las etiquetas que representan estas modificaciones son $<$ Modifica $>$, $<$ Deroga $>$, $<$ Añade $>$ y $<$ Anula $>$.

Estas etiquetas contienen una serie de atributos para indicar qué parte de qué cuerpo legal se está modificando y en qué fecha. En el caso de las sustituciones y las incorporaciones, dentro de la etiqueta se incluye el nuevo texto o el texto que se va añadir.

\subsection{Módulo de base de datos XML}

Se trata del módulo que se encarga de guardar en una colección todos los documentos XML que representan los cuerpos legales; es decir, una vez que un cuerpo legal se ha transformado, con el módulo de edición, en un documento XML que sigue el DTD diseñado, se guarda en la base datos para que el sistema pueda utilizarlo.

Un aspecto muy importante es la utilización de una base de datos nativa XML, la cual se ha empleado principalmente por dos razones: $a$ ) no se necesita mapear el documento XML a una estructura de datos concreta para guardarlo en una base de datos relacional; $b$ ) todas las bases de datos relacionales están centradas en los datos (data-centric databases), pues lo que almacenan en sus campos son datos atómicos, mientras que una base de datos nativa XML no tiene campos y no almacena datos atómicos, sino documentos XML completos.

Un aspecto importante de estas bases de datos es que no utilizan SQL como lenguaje de consulta; en lugar de ello emplean XPATH para seleccionar y hacer re- 
ferencia a texto, elementos, atributos y cualquier otra información contenida dentro de un documento XML.

\subsection{Módulo de consolidación del derecho}

Es el encargado de recuperar el estado de un cuerpo legal en una fecha determinada. Para ello el módulo va procesando el documento XML (que representa el cuerpo legal a mostrar) y al mismo tiempo va creando un nuevo documento XML virtual con el estado del cuerpo legal en esa fecha.

El funcionamiento del módulo es el siguiente:

Primero comprueba que el cuerpo legal esté publicado y en vigor en la fecha que se quiere mostrar.

Después lee la metainformación del documento, y entonces comprueba si ha habido modificaciones del cuerpo legal desde la fecha de publicación hasta la fecha en la que se quiere buscar. Si las ha habido, las guarda en una estructura para su posterior tratamiento.

Entonces va procesando los artículos (apartados y enunciados), que componen el cuerpo legal, uno por uno, comprobando cuáles sufren alguna modificación.

- Si han sufrido alguna modificación, entonces los trata según el tipo de esta. Cuando es una sustitución, va al cuerpo legal modificante, recupera el nuevo texto y entonces guarda en el nuevo documento XML el artículo con el contenido cambiado. Si se trata de una derogación, en el documento nuevo solo guarda el título del artículo junto con unos atributos que indican que ha sido derogado, cuándo y por quién; de esta forma el usuario al consultarlo no ve el contenido pero observa que esa parte ha sido derogada. Si es una anulación, funciona igual que en el caso de una derogación, es decir, en el nuevo documento solo se incluye el título del artículo junto con unos atributos que indican que ha sido anulado, cuándo y la orden judicial que lo ha anulado.

- Si no han sufrido ninguna modificación, se guardan tal cual en el nuevo documento XML.

Una vez procesados los artículos, procesa las incorporaciones que ha tenido el cuerpo legal y que están registradas en la metainformación. Para ello el sistema va al cuerpo legal modificante, recupera la parte que se añade al cuerpo legal y se incluye en la posición correcta.

Este mismo proceso se realiza también para las disposiciones (adicionales, transitorias, derogatorias y finales).

$\mathrm{Al}$ final del proceso se dispone de un nuevo documento XML con los cambios que otros cuerpos legales (u órdenes judiciales) le han realizado. Este nuevo documento XML es un documento "virtual", es decir, no se crean versiones de los 
documentos como hacen algunos de los proyectos europeos (como ocurre en el Proyecto Norma de Italia) (Palmirani, Brighi y Massini, 2004).

El beneficio de utilizar versiones reside en que permitirían una extracción más rápida porque solo habría que acceder a la versión que se tiene guardada del cuerpo legal en la fecha que se está buscando. Por el contrario, tienen muchos inconvenientes, entre los que destaca que, si en el proceso de inserción de un grupo de cuerpos legales se nos olvida introducir una modificación, habría que retocar después los documentos XML de todas las versiones posteriores. Cuando sucede esto en el Proyecto Norma estos cambios son realizados a mano por informáticos que conocen muy bien la estructura que utilizan.

Una vez que el proceso ha finalizado, el nuevo documento XML virtual se transforma utilizando XSLT para mostrarlo en la página web.

Vamos a ver el funcionamiento siguiendo un ejemplo:

Si consultamos en la fecha 15 de septiembre de 1995 el Real Decreto 1451/1983 (figura 5) comprobamos que nos aparece el cuerpo legal en su estado original (figura 6).

Si después cambiamos la fecha de consulta al 15 de septiembre de 2001, el resultado es el que se ve en la figura 7, donde podemos observar que el apartado 1 del artículo 7 ha sido modificado por un cuerpo legal posterior, el Real Decreto 4/1999 (exactamente por el enunciado $1 .^{\circ}$ del apartado 1 del artículo 1 en la fecha 27/01/1999).

Si volvemos a cambiar la fecha al 5 de octubre de 2007, el resultado es el que aparece en la figura 8, en la que vemos que el apartado 1 del artículo 7 está modificado por el RD170/2004, y además el artículo 8 también lo está.

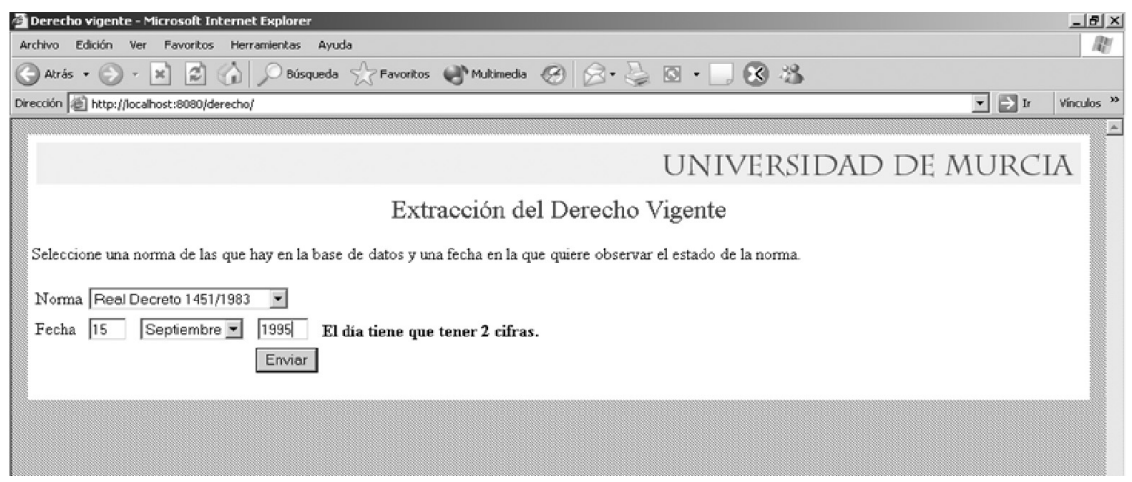

Figura 5. Pantalla inicial del sistema.

Scire. $15: 1$ (en.-jun. 2009) 133-147. ISSN 1135-3716. 


\section{UNIVERSIDAD DE MURCIA}

Extracción del Derecho Vigente
Norma un:um:RD1451/1983 en la fecha 1509/1995

Fecha de publicación: 04/06/1983

Fecha de entrada en vigor: 05/06/1983

Real Decreto $1451 / 1983$, de 11 de mayo, por el que en cumplimiento de lo previsto en la Ley $13 / 1982$, de 7 de abril, se regula el empleo selectivo y las medidas de fomento del empleo de los trabajadores minusválidos.

El Gobierno entiende que es preciso realizar un esfuerzo económico $y$ de solidaridad social para hacer efectivo respecto de los minusválidos el derecho al trabajo que para todos los españoles, sin discriminación, reconoce y garantiza el articulo 35 de la Constitución Española

Por otra parte, el Gobierno en coherencia con el espiritu que inspira la Ley 13/1982, de 7 de abril, de Integración social de los Minusvaslidos, considera que la integraciôn laboral de éstos debe realizarse fundamentalmente a través del sistema ordinario de trabajo y anuncia que a tal fin ira enc aminada la politica de empleo que desarrallará en favor de los minusválidios.

Pues bièn, favorecer la incorporación de los minusvalidos, tanto originarios como sobrevenidos, a puestos de trabajo en la Empresa ordinaria es la que sie persigue con las medidas que se contemplan en el presente Real Decreta que se dicta tanto para dar cumplimiento al mandato legislativo que se contiene en el articulo 40 de la Ley de Integración Social de los Minusvalidos respecto del empleo selectivo, cuanto para introducir determinadas modificaciones en las medidas de fomento del empleo de los minusválidos que hasta ahor venian rigiendo $\mathrm{y}$ al propio tiempo, aprovechando estas circunstancias, para proceder a la unificación de ambos tipos de medidas en una sólà đispósición.

CAPITULO II.- Medidas de fomento del empleo.

Articulo 7.

Las Empresas que contraten por tiempo indefinido y en jomada completa a trabajadores minusvalidos, tencrán derecho a una subvención de $500,000 \mathrm{ptas}$, por cada contrato de trabajo celebrado y a bonificaciones en las cuotas empresariales de la Seguridad Social, incluidas las de accidente de trabajo y enfermedad profesional y las cuotas de recaudación conjunta, en las siguientes cuantias:

a) Setenta por ciento por cada trabajador minusválido contrat ado menor de 45 años.

b) Noventa por ciento por cada trabajador minusvallido contratado mayor de 45 años.

Las Cooperativas de Trabajo Asociado que incorporen a trabajadores minusválidos camo socias, tendrán derecho a los beneficios establecidos en el numero anterior.

Tanto las subvenciones como las bonificaciones serän incompatibles con las que en su caso, otorgue la Unidad Administradora del Fondo Nacional de Prótección al Trabajo y cón cualquier otra medida de fomentó del empleo, salvó lo dispuesto en el articuló 12

Artículo 8.

Las Empresas deberắn solicitar los trabajadores minuswálidos de la cornespondiente oficina de Empleo, con descripción detallada de los puestos a cubri, caracteristicas técnicas de los mismos, asi como capacidad que debe tener el trabajador para cubrir dicho puesto.

La presentación del cantrato que en modelo oficial y por cuacruplicado ejemplar, acompañado de la solicitud de alta en el régimen correspondiente de la Seguridad Social y del Certificado de minusvalia, expedido por el orqanismo competente, surtirá los efectos de correspondiente de la Seguridad Social y del Certificado de minusvalia, expedido por

solicitud de las subvenciones y bonificaciones a que hace referencia el artículo anterior.

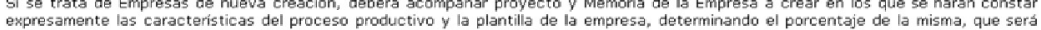
cubierto por minusválidos que no podrá exceder del $51 \%$, porcentaje máximo que no se tendrá en cuenta si la plantilla va a esta integrada únicamente por el trabajador minusválido que se contrata.

El INEM solicitará, además, los siquientes informes:

-De las equipos multiprofesianales, sobre la adecuación del puesto de trabajo a la minusvalía de los trabajadores que, teniendo tal condición, se encuentren inscritas en la Oficina de Emplea.

Figura 6. Estado original del RD 1451/1983.

\subsection{Módulo de búsqueda}

Este módulo permite realizar búsquedas sobre los cuerpos legales incluidos en la base de datos, según diferentes criterios, como rango, número, fecha de aprobación, fecha de publicación, fecha de entrada en vigor...

Actualmente se está investigando para mejorar este módulo, incluyéndole búsquedas semánticas para facilitar la labor de recuperar un cuerpo legal aplicable a un caso, que es el punto más importante de la investigación presente (aunque hoy por hoy no existen resultados).

Scire. $15: 1$ (en.-jun. 2009) 133-147. ISSN 1135-3716. 
Artículo 7.

1. Las empresas que contraten por tiempo indefinido $y$ a jornada completa a trabajadores minusválidos, tendrán derecho a una cubuencion de 650 nחn nesetac nor cada contrato de trabajo celebrado, y durante su vigencia, a bonificaciones en las cuotas Modficacion Reaizada por'urnim: Ro4/1999@1.1.1'enla jidas las de accidente de trabajo $y$ enfermedad profesional y las cuotas de recaudación fecha' $27101 / 1999$

a) 70 por 100 por cada trabajador minusválido contratado menor de cuarenta y cinco años.

b) 90 por 100 por cada trabajador minusválido contratado mayor de cuarenta y cinco años.

Cuando el contrato por tiempo indefinido se concierte a tiempo parcial, se mantendrán las bonificaciones en las cuotas descritas en el apartado anterior, si bien la subvención de 650.000 pesetas se reducirá proporcionalmente a la jornada pactadas.

Las Cooperativas de Trabajo Asociado que incorporen a trabajadores minusválidos como sucios, tendrán derecho a los beneficios establecidos en el número anterior.

Tanto las subvenciones como las bonificaciones serán incompatibles con las que en su caso, otorgue la Unidad Administradora del Fondo Nacional de Protección al Trabajo y con cualquier otra medida de fomento del empleo, salvo lo dispuesto en el artículo 12.

Artículo 8.

Las Empresas deberán solicitar los trabajadores minusválidos de la correspondiente oficina de Empleo, con descripción detallada de los puestos a cubrir, características técnicas de los mismos, así como capacidad que debe tener el trabajador para cubrir dicho puesto.

La presentación del contrato que en modelo oficial y por cuadruplicado ejemplar, acompañado de la solicitud de alta en el régimen correspondiente de la Seguridad Social y del Certificado de minusvalía, expedido por el organismo competente, surtirá los efectos de solicitud de las subvenciones y bonificaciones a que hace referencia el artículo anterior.

Si se trata de Empresas de nueva creación, deberá acompañar provecto y Memoria de la Empresa a crear en los que $5 e$ harán constar expresamente las características del proceso productivo y la plantilla de la empresa, determinando el porcentaje de la misma, que será cubierto por minusválidos que no podrá exceder del $51 \%$, porcentaje máximo que no se tendrá en cuenta si la plantilla va a estar integrada únicamente por el trabajador minusválido que se contrata.

\section{Figura 7. Estado del RD 1451/1983 el 15/09/2001.}

GAPITULO II.- Medidas de fomento del empleo.

Articulo 7.

1. 1. Las empresas que contraten por tiempo indefinido y a jomada completa a trabajadores minusválidos tendrán derecho a una

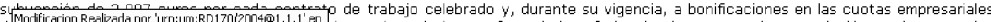

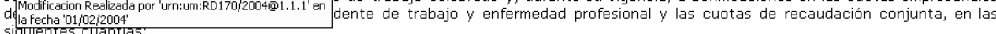

a) $70 \%$ por cada trabajador minusuálida contratado menor de 45 años. En el casa de contratación de mujeres minusuálidas, dicho porcentaje será del 9096

b) $90 \%$ por cada trabajador minusválido contratado de 45 o más años. En el caso de contratación de mujeres minusválidas, dicho porcențje será del $100 \%$.

Cuando el contrato por tiempo indefinido se concierte a tiempo parcial, se mentendrán las bonificaciones en las cuotas descritas en los párrafos anteriores, si bien la subvención de 3.907 euros se reducirá proporcionalmente a la jornada pactada.

Las Cooperativas de Trabajo Asociado que incorporen a trabajadores minusválidos como socios, tendrán derecho a los beneficios establecidos en el número anterio

Tanto las subvenciones como las bonificaciones serán incompatibles con las que en su caso, otorgue la Unidad Administradora del Fondo Nacional de Protección al Trabajo y con cualquier otra medida de fomento del emoleo, salvo lo dispuesto en el articulo 12.

1 Artículo 8.

Las empresas deberán solicitar los trabajadores minusválidos de la correspondiente aficina de empleo, con descripción detallada de los puestos que se quieran cubrir, sus características técnicas, así como capacidad que debe tener el trabajador para cubrir dicha puesto. La presentación del contrato en modelo oficial $y$ por ejemplar cuadruplicado, acompañado de la solicitud de alta en el régimen correspondiente de la Seguridad Social y del certificado que acredite el grado de minusvalia, expedido por el organismo competente surtirá los efectos de solicitud de las subvenciones y bonificaciones a que hace referencia el artículo anterior.

El servicio pública de empleo competente solicitará el informe de los equipos multiprofesionales sobre la adecuación del puesto de trabajo a la minusvalía de los trabajadores que, teniendo acreditada tal circunstancia, se encuentren inscritos en la oficina de empleo.

Figura 8. Estado del RD 1451/1983 el 5/10/2007. 


\subsection{Módulo web para el acceso uniforme a la información}

Módulo que se encarga de mostrar todo a través de la Web, es decir, de hacer que tanto la aplicación para la edición de cuerpos legales como el buscador y la aplicación para la recuperación del estado de un cuerpo legal en una fecha determinada sean accesibles vía web.

\section{Conclusiones y trabajos futuros}

Con este proyecto se facilita la labor de un usuario cuando necesita recuperar el estado de un cuerpo legal en una fecha concreta, ya que se accede automáticamente a todos los cuerpos legales que modifican a uno concreto, y así él no tiene que realizarlo manualmente. De esta forma, el usuario se ahorra mucho esfuerzo y una gran cantidad de horas, y se evita además los posibles fallos que se podrían ocasionar si lo hiciese a mano y no tuviese en cuenta un cuerpo legal que modificaba al que quería consultar; por ejemplo, si no se acordase de consultar una ley que derogaba un artículo del cuerpo legal que desea consultar y toma ese artículo como válido.

Una de las perspectivas de desarrollo de la investigación consiste en integrar el sistema creado en el contexto de un programa de ayuda a la localización de la información relevante mucho más completo. No hay que olvidar que la utilidad principal de la determinación de la versión en vigor de un texto jurídico se muestra en la búsqueda del cuerpo legal aplicable a un caso concreto.

La peculiaridad de este tipo de búsqueda viene dada por el hecho de que la información requerida está integrada por cuerpos legales que son relevantes por su aplicabilidad a una determinada situación (por ejemplo, se buscan los cuerpos legales que son aplicables a un contrato de arrendamiento).

El planteamiento y la resolución de un problema jurídico, es decir, un problema cuya solución requiere una decisión fundada en derecho, pasa por la selección del cuerpo legal que se refiere al caso planteado asignándole un específico efecto jurídico. La determinación del cuerpo legal aplicable es una operación esencial en el procedimiento de aplicación del derecho; se trata de una operación compleja, puesto que los descriptivos (términos de búsqueda) requeridos para discriminar el resultado pretendido (el cuerpo legal aplicable) dentro del ámbito de referencia (el ordenamiento jurídico en su conjunto) han de obtenerse a través de la observación de las características relevantes del problema planteado.

De este modo, ha de atenderse al tipo jurídico al que pertenece la situación planteada (por ejemplo, si es un contrato, compraventa, arrendamiento, depósito, etcétera; si es un delito, homicidio, robo, estafa...). También habrá que tener en cuenta la condición de los sujetos que intervienen (si son comerciantes, si son funcionarios, etcétera) y la vinculación de los eventos o de sus participantes a un te-

Scire. 15 : 1 (en.-jun. 2009) 133-147. ISSN 1135-3716. 
rritorio (determinando de este modo la aplicación de normas de conflicto internacionales o interregionales). Pero, de todas las circunstancias relevantes en la búsqueda del cuerpo legal aplicable, lo que ahora nos interesa destacar es el papel que desempeña en esta tarea tanto la fecha de producción de los acontecimientos determinantes del problema como aquella en que los cuerpos legales son aplicados para obtener la solución jurídica del problema.

A la hora de generar el programa de ayuda a la búsqueda de la disposición normativa aplicable hay que tener en cuenta que el sistema tendrá que operar con los siguientes datos: $a$ ) primero con la fecha en que se pide la solución del caso, seleccionando únicamente los cuerpos legales que son normas jurídicas en esa fecha; $b$ ) en segundo lugar, con la fecha en que se ha generado el problema, que es uno de los factores (junto con la materia, los sujetos o el territorio) que permitirán discriminar cuáles de esas normas jurídicas son aplicables al caso.

Si resulta que, según esta última fecha, el cuerpo legal aplicable es una disposición de derecho transitorio que remite a otros cuerpos legales que ya han sido derogados, el programa debe estar en condiciones de encontrar la versión de ese cuerpo legal en la fecha señalada. Precisamente en este aspecto de la búsqueda cobra especial interés el poder disponer de las diversas versiones que ha tenido un cuerpo legal tras sus sucesivas modificaciones. Debido a esto, un sistema como el descrito en este artículo es un buen punto de partida para el desarrollo de un sistema de búsqueda del cuerpo legal aplicable a un caso concreto.

En todo caso, este es tan solo uno de los aspectos - muy importante, sin duda - que deben ser cuidados en la creación del sistema de búsqueda que se pretende desarrollar. Hay muchos otros que han de tenerse en cuenta, tales como la especificación de los intervalos de subsunción de cada cuerpo legal, la elaboración de un catálogo de materias jurídicas que permita establecer correspondencias automáticas entre los casos planteados y los cuerpos legales aplicables, etcétera.

\section{Referencias}

Biagioli, C.; Francesconi, E.; Spinosa, P.; Taddei, M. (2003). The NIR Project: standards and tools for legislative drafting and legal document Web publication. Edimburgo: ICAIL, 2003.

Biagioli, C.; Francesconi, E.; Spinosa, P. (2005). A legal drafting environment based on formal and semantic XML standards. Boloni: ICAIL, 2005.

Bourret, R. (2008). XML and databases: a discussion of the relationship between XML and databases. Includes a discussion of native XML databases. http://www.rpbourret.com/ xml/XMLAndDatabases.htm (2008-06).

Hernández Marín, R. (2002). Introducción a la teoría de la norma jurídica. Madrid, Barcelona: Marcial Pons, 2002. 442 y ss.

Scire. $15: 1$ (en.-jun. 2009) 133-147. ISSN 1135-3716. 
Palmirani, M.; Brighi, R.; Massini, M. (2003). Automated extraction of normative references in legal texts. Edimburgo: ICAIL, 2003.

Palmirani, M.; Brighi, R.; Massini, M. (2004). Processing Normative References on the Basis of Natural Language Questions. // NLIS'04: $4^{\text {th }}$ International Workshop on Natural Language and Information Systems, DEXA Workshops, 2004. 9-12.

Moens, M. (2004). XML retrieval models for legislation. Berlín: Jurix, 2004.

Recibido: 2008-06-25. Aceptado: 2008-07-01

Scire. $15: 1$ (en.-jun. 2009) 133-147. ISSN 1135-3716. 